\title{
Evaluación de Bienestar Psicológico en habitantes del Estado de Hidalgo con el apoyo de las TICs
}

\author{
Measurement of Psychological Well-being among inhabitants of Hidalgo State with \\ the ICT support
}
Ernesto Cotonieto Martínez ${ }^{a}$, Marcos Galván ${ }^{b}$, Andrómeda Ivette Valencia Ortiz ${ }^{c}$, Guadalupe López-Rodríguez, ${ }^{d}$

\begin{abstract}
:
Background: The information and communications technologies (ICT) facilities the access to information and allow spread the knowledge generated in all the science fields. Method: A cross-sectional study was conducted on 50 inhabitants of Hidalgo State who were contacted by WhatsApp and Facebook. The objective was measuring their Psychological Well-Being with the ICT support. The Positive Psychological Functioning (PPF) Scale was digitalized in Google Forms and used to collect the data, which were analysed using SPSS Version 24 software. Results: The scale were completed by of 28 male and 22 female between 19 and 54 years old. The sample mean age was $26.4(\mathrm{SD}=$ 6.2). Scores close to the theoretical average were found. Differences between mean scores according to sex, marital status or academic level were not found. The period of time with the highest frequency of reception of responses was between 12: 00-18: 00 hours. Conclusions: The results suggest an absence of psychological problems associate to selfesteem, optimism, resilience or environment domain. Positive (economic cost reduction) and negative (Not being able to observe attitudes during the application) aspects about the psychometric measurement supported by ICT were identified. However, this study should be complemented with more research.
\end{abstract}

Keywords:

Scale, Measurement, Psychology, Technology, Communication.

\section{Resumen:}

Antecedentes: Las tecnologías de la información y las communicaciones (TICs) facilitan el acceso a la información y permite difundir el conocimiento generado en todos los campos de la ciencia. Método: se realizó un estudio transversal en 50 habitantes del estado de Hidalgo que fueron contactados por WhatsApp y Facebook. El objetivo fue medir su bienestar psicológico con el apoyo de las TICs. Se utilizó la Escala de Funcionamiento Psicológico Positivo (FPP), la cual fue digitalizada en Google Forms para recopilar los datos que posteriormente fueron analizados con el software SPSS Versión 24. Resultados: La escala fue completada por 28 hombres y 22 mujeres entre 19 y 54 años. La media de edad fue 26.4 años $(\mathrm{DE}=6.2)$. Se encontraron puntajes cercanos a la media teórica. No se encontraron diferencias entre las puntuaciones según el sexo, estado civil o nivel académico. El período de tiempo con la mayor frecuencia de recepción de respuestas fue entre las 12: 00-18: 00 horas. Conclusiones: Los resultados sugieren una ausencia de problemas psicológicos asociados a autoestima, optimismo, resiliencia o dominio del entorno. Se identificaron aspectos positivos (reducción de costos económicos) y negativos (no poder observar actitudes durante la aplicación) sobre la evaluación psicométrica con el apoyo de las TICs. Sin embargo, los resultados de este estudio deben ser complementados con otras investigaciones.

Palabras Clave:

Escala, Medición, Psicología, Tecnología, Comunicación.

${ }^{a}$ Autor de Correspondencia, Universidad Autónoma del Estado de Hidalgo, Instituto de Ciencias de la Salud,

ORCID: https://orcid.org/0000-0001-5639-5898, Email: cotonietoe@gmail.com

${ }^{\text {b}}$ Universidad Autónoma del Estado de Hidalgo, Instituto de Ciencias de la Salud, ORCID: https://orcid.org/0000-0002-3254-4470

Email: mgalvan73@hotmail.com

${ }^{c}$ Universidad Autónoma del Estado de Hidalgo, Instituto de Ciencias de la Salud. ORCID: https://orcid.org/0000-0001-9664-1993 Email: andromeda_valencia@uaeh.edu.mx

dUniversidad Autónoma del Estado de Hidalgo, Instituto de Ciencias de la Salud. Email: glopez@uaeh.edu.mx 


\section{Introducción}

Las Tecnologías de la Información y Comunicación (TICs) son herramientas que permiten optimizar el acceso a la información; a través de ellas es posible generar, difundir y optimizar el conocimiento a bajo costo económico ${ }^{(1)}$.

Las TICs han impactado todas las áreas de la vida de las personas en términos de comunicación, finanzas, política, mercado laboral, y por supuesto, salud; modificando constantemente la forma en cómo nos relacionamos en el entorno laboral, familiar y educativo (2).

En México, el $74.3 \%$ de personas entre 25 y 34 años y el $85.5 \%$ del grupo de edad de 12 a 17 años reportan hacer uso de Internet ${ }^{(1)}$, es decir, la incorporación de las TICs es un área de oportunidad para la investigación científica en el contexto actual de la población mexicana, considerando su habilidades para la familiaridad con tecnologías que requieren de acceso a Internet.

En el Estado de Hidalgo específicamente, el uso de TICs para la investigación es un área de oportunidad a corto, mediano y largo plazo ya que de acuerdo con cifras del INEGI, para el 2016 el $66.9 \%$ de los hogares en Pachuca de Soto ya contaban con acceso a Internet. Considerando que el promedio nacional fue de $47 \%$, Hidalgo cuenta con prácticamente 20 puntos porcentuales por encima de la media. Además, el porcentaje de los Hidalguenses con acceso a Internet fijo y móvil es de $77.9 \%$, frente al promedio nacional de $59.5 \%{ }^{(2)}$.

Estas herramientas digitales han sido utilizadas para el desarrollo y generación de conocimientos en el área de la Psicología tanto en investigación experimental como en no experimental para alcanzar objetivos como la obtención de perfiles de personalidad (3) 0 la implementación de programas de intervención psicológica vía Internet ${ }^{(4)}$.

En cualquier diseño de investigación en psicología que implique el llenado de auto-reportes de opción múltiple por parte de los participantes, el uso de las TICs tiene algunas ventajas frente al método tradicional de lápizpapel: reducción de costos, tiempo y errores durante la transcripción de respuestas; y aunque no se han encontrado diferencias en cuanto al tiempo dedicado a responder las pruebas psicométricas, la reducción de recursos humanos y materiales destinados a la recolección y análisis de datos ${ }^{(5,6)}$.

\section{Objetivo}

Evaluar el bienestar psicológico de una muestra de pobladores del Estado de Hidalgo mediante de la aplicación de un instrumento psicométrico con el apoyo de las TICs.

\section{Materiales y métodos}

Se utilizó la Escala de Funcionamiento Psicológico Positivo (FPP), desarrollada en España por Merino y Privado en el año $2007^{(7)}$ para evaluar el bienestar psicológico de las personas. Consta de 33 reactivos tipo Likert, cada uno con opciones de respuesta desde "Completamente en desacuerdo" hasta "Completamente de acuerdo". Se encuentran agrupados en once factores de primer orden (tres reactivos por cada factor) y uno general de segundo orden.

La validación Mexicana fue realizada en 2015 por Merino, Privado y Gracia ${ }^{(8)}$, con una muestra conformada por 184 estudiantes de psicología pertenecientes a la Universidad Nacional Autónoma de México (UNAM), obteniendo $\alpha$ desde 0.52 hasta 0.84 .

Participantes. En la aplicación de la escala FPP participaron 50 personas de entre 19 y 54 años de edad, los criterios de inclusión fueron: i) contar con WhatsApp - Facebook en algún dispositivo para poder recibir la liga del formulario, ii) acceso a Internet fijo o móvil, iii) ser mayor de edad, iv) vivir en el estado de Hidalgo y v) leer el consentimiento informado. Se trató de un muestreo no probabilístico por bola de nieve, ya que se invitó a participar en el muestreo vía WhatsApp y Facebook.

Posteriormente se solicitó su participación para alcanzar un total de 50. Una vez leído y aceptado lo planteado en el consentimiento informado, los participantes respondieron un breve apartado de preguntas sociodemográficas, y la escala FPP en modalidad virtual a través de un formulario creado en Google Drive. Después de haber alcanzado un tamaño de 50 participantes, ideal para garantizar que se conserva la estructura factorial de la escala ${ }^{(9)}$, la liga fue invalidada, enseguida se procedió al análisis de la información recopilada, y finalmente a la devolución de los resultados a quienes lo solicitaron (Figura 1).

Formulario de Google Drive. Es una herramienta de Google que permite crear, enviar y evaluar cuestionarios de manera muy rápida y sencilla. Durante la creación, despliega una serie de opciones a fin de configurarlo de 
acuerdo a las necesidades del usuario, por ejemplo: permite configurar el tipo de respuesta solicitada para cada pregunta (selección múltiple, casillas de verificación, desplegable o respuesta corta), determinar claves de respuestas, añadir comentarios sobre las respuestas, y elegir qué ven las personas después de enviar sus respuestas: incorrectas, correctas o puntuaciones crudas.

Los formularios de Google Drive también dan alternativas en cuanto a la evaluación de respuestas del cuestionario, cuenta con resúmenes automáticos que pueden incluir: preguntas en las que frecuentemente fallan, gráficos con respuestas correctas, así como promedio, media e intervalo de puntuaciones. Además, si el usuario lo requiere, permite evaluar las respuestas individuales y las preguntas de forma independiente, y finalmente es posible enviar los resultados por correo electrónico. Aquí se puede conocer más detalles sobre los

formularios:

https://www.google.com/intl/es/forms/about/

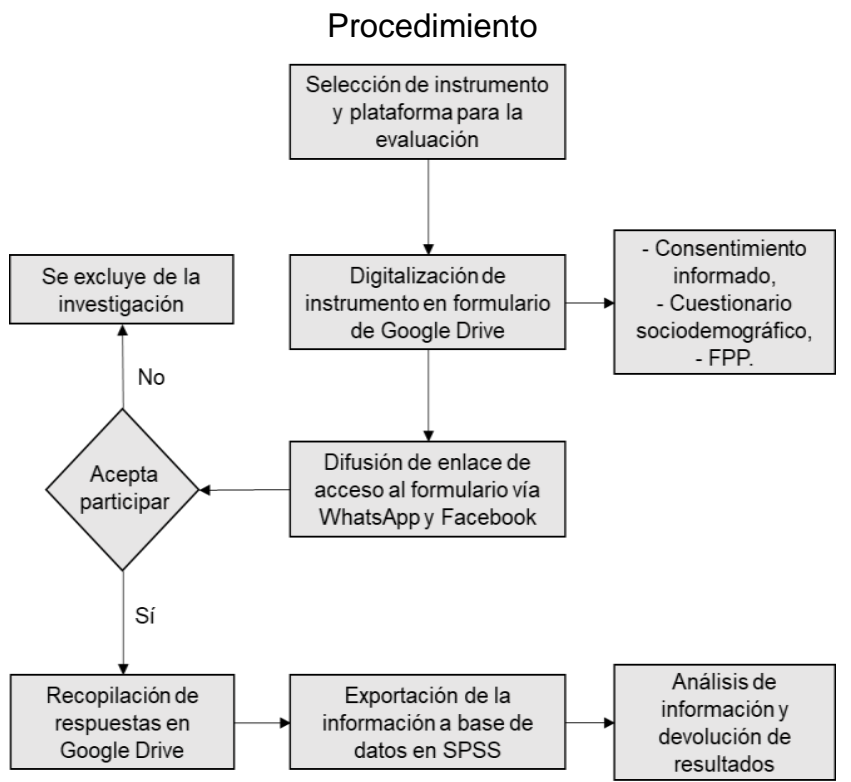

Figura1. Procedimiento para la aplicación de escala de Funcionamiento Psicológico Positivo (FPP) a través de Google Drive.

\section{Análisis estadístico}

Todos los análisis estadísticos se realizaron en software SPSS versión 24. Se esperaba que la distribución de la muestra se comportara con normalidad ya que la investigación se llevó a cabo en población general y no se trataba de población con características clínicas, por lo que se aplicó la prueba Shapiro-Wilk y se verificó el supuesto de normalidad, respecto al puntaje general obtenido en la escala ( $p=0.099)$.

Con base en el resultado de la normalidad y el tamaño de la muestra $(\mathrm{N} \geq 30)$ se utilizó la prueba t- Student para muestras independientes, tal y como lo sugiere la literatura ${ }^{(10)}$, con la finalidad de comparar los puntajes obtenidos de la escala FPP en relación con grupos de sexo, grado de estudios y estado civil.

\section{Resultados}

Resultados del proceso de aplicación. El enlace de acceso al formulario Google Drive comenzó a enviarse a las 6:00 am del día 24 de octubre del 2017, y se mantuvo activo hasta las 00:00 horas del 25 de octubre del mismo año. Las respuestas y la hora exacta de recepción fueron registras de forma automática en la plataforma de Google Drive; a partir de ello, es posible observar en la Figura 2 que el periodo en que se recibió mayor cantidad de respuestas fue entre las 12:00 pm y las 5:59 pm.

\section{Horario de recepción}

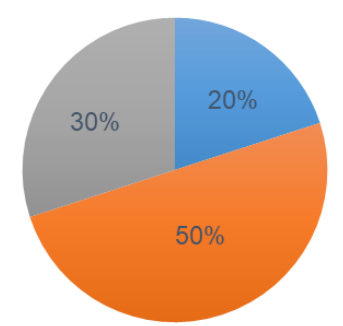

- Entre las 6:00 y 11:59 am_ Entre las $12: 00$ y las $5: 59$ pm - Entre las 6:00 y las 11:59 pm

Figura 2. Periodos de recepción de respuestas en Google Drive.

Respecto a la devolución de los resultados, sólo se recibió una solicitud de retroalimentación y el resto se abstuvieron de realizar la petición.

Se obtuvieron respuestas de 28 hombres y 22 mujeres $(\mathrm{N}=50)$, con una media de edad de 26.4 años $(\mathrm{sd}=6.2)$. La mayoría de los participantes reportaron estar solteras $(68 \%)$ y una tercera parte $(32 \%)$ tiene un nivel educativo igual 0 menor a Preparatoria (Tabla 1). 
Tabla 1. Caracteristicas sociodemográficas de los participantes

\begin{tabular}{lccc}
\hline Características & Hombres & Mujeres & Total \\
\hline & $\mathrm{N}=28$ & $\mathrm{~N}=22$ & $\mathrm{~N}=50$ \\
& $56 \%$ & $44 \%$ & $100 \%$ \\
Edad & 26.46 & 26.32 & 26.4 \\
Media & 7.11 & 4.98 & 6.20 \\
$\begin{array}{l}\text { Desviación estándar } \\
\text { Estado civil }\end{array} \quad 20$ & 14 & $68 \%$ \\
$\quad \begin{array}{l}\text { Solteras } \\
\quad \text { Casadas }\end{array}$ & 8 & 8 & $32 \%$ \\
$\quad$ Grado de estudios & 9 & 7 & $32 \%$ \\
$\quad$ Igual o menor a preparatoria & 19 & 15 & $68 \%$ \\
\hline$\quad$ gual o mayor a licenciatura & & &
\end{tabular}

La población obtuvo puntajes de funcionamiento psicológico levemente por encima de la media esperada, tanto en los factores de primer orden como en el de segundo orden (Tabla 2).

Tabla 2. Medias esperadas y medias obtenidas, por cada factor de la Escala de Funcionamiento Psicológico Positivo.

\begin{tabular}{lccc}
\hline Factor & $\begin{array}{c}\text { Media } \\
\text { esperada }\end{array}$ & $\begin{array}{c}\text { Media } \\
\text { obtenida }\end{array}$ & $\begin{array}{c}\text { Desviació } \\
\mathbf{n} \\
\text { estándar }\end{array}$ \\
\hline Autoestima & 9 & 10.62 & 1.95 \\
Resiliencia & 9 & 12.58 & 2.05 \\
Optimismo & 9 & 12.9 & 2.07 \\
Creatividad & 9 & 12.4 & 1.99 \\
Autonomía & 9 & 12.76 & 1.9 \\
Dominio del entorno & 9 & 10.34 & 2.03 \\
Vitalidad & 9 & 12.28 & 2.29 \\
Propósito vital & 9 & 13.34 & 1.83 \\
Humor & 9 & 13.18 & 1.79 \\
Disfrute & 9 & 12.62 & 1.56 \\
Curiosidad & 9 & 13.14 & 1.7 \\
Funcionamiento psicológico & 99 & 136.16 & 13.68 \\
positivo & & & \\
\hline
\end{tabular}

No se encontraron diferencias estadísticamente significativas entre las medias obtenidas por los hombres en comparación con las mujeres respecto al puntaje general de funcionamiento psicológico positivo $(t=-0.694, g l=48, p=0.491)$ o alguno de los factores de la prueba (Tabla 3 ).

Tabla 3. Medias obtenidas por hombres y mujeres en cada factorde la Escala de Funcionamiento Psicológico Positivo.

\begin{tabular}{lcc}
\hline \multirow{2}{*}{ Factor } & \multicolumn{2}{c}{ Medias } \\
& Mujeres & Hombres \\
\hline Autoestima & 11.09 & 10.25 \\
Resiliencia & 13.05 & 12.21 \\
Optimismo & 12.82 & 12.96 \\
Creatividad & 11.86 & 12.82 \\
Autonomía & 12.55 & 12.93 \\
Dominio del entorno & 9.73 & 10.82 \\
Vitalidad & 12.05 & 12.46 \\
Propósito vital & 13.32 & 13.36 \\
Humor & 12.82 & 13.46 \\
Disfrute & 12.50 & 12.71 \\
Curiosidad & 12.86 & 13.36 \\
Funcionamiento psicológico positivo & 134.64 & 137.36 \\
\hline
\end{tabular}

En cuanto al estado civil, tampoco se encontraron diferencias estadísticamente significativas $(\mathrm{t}=-0.827, \mathrm{gl}=$ $48, p=0.413$ ), ni en relación al nivel de estudios ( $t=$ -
0.122, $g l=48, p=0.983)$; es decir, no son factores determinantes en los resultados respecto al funcionamiento psicológico en general; y en ninguno de los factores de primer orden.

\section{Discusión}

Los resultados indican que las personas residentes del Estado de Hidalgo, que formaron parte de la muestra de esta investigación, tienen niveles de bienestar psicológico dentro de los esperado, incluso se encontraron puntajes levemente por encima de la media teórica del instrumento utilizado, así como desviaciones estándar mínimas. Estos resultados sugieren una ausencia de problemas psicológicos en cuanto a indicadores de autoestima, optimismo, resiliencia o dominio del entorno, entre otros.

Lo cual era esperado ya que la aplicación se llevó a cabo con personas clínicamente sanas, motivo por el cual, posiblemente, se obtuvieron resultados cercanos a la media teórica del instrumento. También se encontró que los puntajes en la escala FPP no varían en función de las variables sociodemográficas evaluadas. Por lo tanto, el bienestar emocional de las personas residentes de la entidad no es diferente si son hombres, mujeres, solteras, casadas, si tienen un nivel educativo básico o superior.

Sin embargo, esta información podría corroborarse llevando a cabo otras investigaciones que incluyan la aplicación de más instrumentos psicométricos que midan otros constructos. El cruce de esta información serviría para corroborar la interacción entre las variables usando pruebas estadísticas de correlación.

Por ejemplo, el estudio de Araujo \& Savigon ${ }^{(11)}$, en el que se aplicó el cuestionario de bienestar psicológico (CAVIAR) junto con la escala de 16 Factores de la personalidad (16 FP), o la investigación de Matalinares y cols. ${ }^{(12)}$, en donde se aplicó la Escala de Bienestar Psicológico (SPWB) y la Escala de Modos de Afrontamiento al Estrés (COPE), y se hicieron pruebas de correlación.

Además, tomando en cuenta que el tipo de muestreo utilizado no permite garantizar la representatividad de la muestra respecto a la población Hidalguense, tampoco es posible generalizar los resultados de la escala FPP ya que hubo un control mínimo sobre la selección de la muestra. Es decir, en el presente estudio se pueden encontrar diversas fuentes de invalidez interna y externa que se pueden fortalecer en futuras implementaciones. 
Las TICs son capaces de crear puentes de comunicación e interacción nunca antes vistos, en definitiva, son una herramienta mediante la cual se facilita el proceso de generación del conocimiento ${ }^{(13)}$, e implica tanto ventajas como desventajas en materia de investigación. La presente investigación no fue la excepción y a partir de nuestra experiencia identificamos algunas implicaciones positivas y negativas de su uso durante la evaluación.

Entre los aspectos negativos se encuentran: Imposibilidad de atender dudas sobre el significado de alguna pregunta específica o la forma adecuada para responder el cuestionario en general. $Y$ aunque al final de la evaluación se agregó un apartado para solicitar un informe de los resultados, aclarar dudas y comentarios, esto no aseguró que los participantes comprendieran todo lo relacionado con la evaluación, lo cual limita para el cumplimiento de los estándares de ética deseables.

De igual forma, se perdió la oportunidad de observar aspectos relacionados con el tiempo de respuesta, actitudes o indicadores de ansiedad, estrés o estado de ánimo durante la aplicación; aspectos pudieron aportar información relevante para complementar la interpretación de los resultados.

Entre los aspectos positivos, podemos mencionar un ahorro del $100 \%$ sobre el costo económico derivado del uso de bolígrafos, papel, tinta o transporte necesario para aplicar el instrumento de manera física. Reducción de recursos humanos y tiempo requerido para el proceso de aplicación, captura de datos y su posterior calificación. Además de la disminución de errores asociados con la captura de respuestas en la paquetería estadística.

Asimismo, fue posible identificar los periodos de tiempo con menor disponibilidad para responder el cuestionario en línea: 1) antes de las 12:00 horas y después de las 18:00. En el primer caso, podría estar relacionado con una mayor atención a otras actividades al inicio del día (académicas o laborales). En el segundo caso, con el cansancio acumulado por dichas actividades al finalizar la jornada y que por lo tanto indispone la participación en actividades "extra", como una evaluación de este tipo.

Esa información puede ser relevante en la difusión de enlaces de acceso a instrumentos de evaluación psicométrica digitalizada. Es decir, se puede optar por hacer invitaciones por primera vez o recordarles a las personas que está pendiente su participación en el periodo de tiempo que identificamos como de mayor disponibilidad, con esto se incrementaría la posibilidad de participación en investigaciones similares.

Adicionalmente, sería importante que futuras investigaciones exploren la percepción de los participantes respecto a una evaluación psicométrica aplicada de manera digital; conocer si validan este tipo de aplicaciones podría ser de gran utilidad en la interpretación de los resultados a obtener.

Otra acción complementaria puede ser la aplicación simultánea de los instrumentos en modalidad papel y digital para identificar si existen diferencias en cuanto a los resultados, la percepción del instrumento o del mismo proceso de evaluación.

\section{Conclusiones}

A manera de conclusión, podemos destacar que los resultados obtenidos a través de esta investigación permitieron: a) describir el bienestar psicológico en habitantes del Estado de Hidalgo y b) identificar los aspectos positivos y negativos de dicha evaluación utilizando un instrumento digitalizado y difundido a través de las TICs.

Este primer acercamiento confirma la importancia de llevar a cabo pruebas piloto ${ }^{(14,15)}$, ya que la información preliminar y el aprendizaje aquí generado ayudará a refinar los procedimientos más adecuados para hacer una futuras evaluaciones de bienestar psicológico ( $u$ otras variables) de manera más precisa, sacando ventaja de las bondades que ofrece el uso de las TICs en investigación.

Por ejemplo, es bien sabido que el elevado costo por insumos para los estudios científicos generalmente limita la acción del investigador, puesto que en ocasiones los recursos asignados para estas actividades es limitado, y sobre todo en aquellos en los que se contempla una $\mathrm{N}$ grande. Al respecto, la plataforma de Google Drive puede ser una alternativa para disminuir la inversión en recursos materiales y humanos, aunque hay que considerar las implicaciones negativas del uso de este tipo de plataformas, especialmente en investigación de variables psicosociales.

Finalmente, considerando las cifras actuales en el Estado de Hidalgo respecto al acceso a Internet, podemos afirmar que el uso de plataformas tecnológicas y su incorporación a la investigación científica es una alternativa viable para quienes requieren generar conocimiento científico en la región. 


\section{Referencias}

1. Instituto Nacional de Estadística y Geografía "Estadísticas a Propósito Del Día Mundial De Internet ( 17 De Mayo )". 2017. Disponible en: http://www.inegi.org.mx/saladeprensa/aproposito /2017/internet2017_Nal.pdf

2. UNESCO. Enfoque Estratégico Sobre Tics En Educación En América Latina $Y$ El Caribe [Internet]. Enfoque Estratégico Sobre Tics En Educación En América Latina Y El Caribe. Chile; 2013. Disponible en: http://www.unesco.org/new/fileadmin/MULTIMED IA/FIELD/Santiago/images/ticsesp.pdf

3. González-Santos L, Mercadillo RE, Graff A, Barrios FA. Versión Computarizada para la aplicación del Listado de Síntomas 90 y del Inventario de Temperamento y Carácter. Salud Mental. 2007; 30: 31-9.

4. Stoddard J, Deelucchi K, Collins N, Stable EP, Augustson E, Lenert L. Smoking Cessation Research via The Internet: A Feasibility Study. Journal of Health Communication; 10: 27-41.

5. Gibbs GR, Friese S, Mangabeira WC. The Use of New Technology in Qualitative Research. Introduction to Issue 3 ( 2 ) of FQS. Technology. 2002;3(2):1-13.

6. Morales-Ramírez A, Escoto MC, García-Lozano RZ, Molinar-Solís JE, Hidalgo-Cortés C. Sistema para la Aplicación de Pruebas Psicológicas vía Web. Acta Universitaria.2012; 22(3): 5-13.

7. Dolores Merino M, Privado J. Positive Psychological Functioning. Evidence for a new construct and its measurement. An Psicol. 2015;31(1):45-54.

8. Merino MD, Privado J, Gracia Z. Validación mexicana de la Escala de Funcionamiento Psicológico Positivo. Perspectivas en torno al estudio del bienestar y su medida. Salud Ment. 2015;38(2):109-15.

9. Paniagua R. Metodología para La Evaluación de una Escala o Instrumento de Medida. Univ Antioquia Fac Nac Salud Pública. 2015;(1):1-5.

10. Sánchez RA. t-Student. Usos y abusos. Revista mexicana de cardiología. 2015; 26(1): 59-61

11. Araujo V. \& Savignon D. Bienestar psicológico en estudiantes repitentes de la Facultad de Psicología de la Universidad de La Habana. Revista Cubana Educación Superior. 2018; 2(1): 178-88.
12. Matalinares ML, Díaz G, Arenas C, Raymundo O, Baca D, Uceda J, \& Yaringaño L. Afrontamiento al estrés y bienestar psicológico en estudiantes universitarios de Lima y Huancayo. Revista IIPSI. 2016; 19(2): 123-43.

13. Agenda I, Forum WE. Digital Media and Society Implications in a Hyperconnected Era. 2016;(January):64.

14. Abu Z, Fracgp H, Mmed PS, Fracgp DM. Research Notes DOING A PILOT STUDY : WHY IS IT ESSENTIAL? 2006;1(2):70-3.

15. van Teijlingen E, Hundley $\mathrm{V}$. The importance of pilot studies. Nurs Stand [Internet]. 2002;16(40):33-6. Disponible en: http://rcnpublishing.com/doi/abs/10.7748/ns2002. 06.16.40.33.c3214 